\title{
Melatonin-Rezeptoragonist erfolgreich bei Non-24
}

\begin{abstract}
_ Weil die endogene Uhr nicht exakt im 24-Stunden-Takt, sondern in einem zirkadianen Rhythmus läuft, kann der endogene Tag länger sein als der Umgebungstag. Wichtigster Taktgeber der Synchronisation der beiden Rhythmen ist das Licht. Dyssynchronie kann zu Schlaf-Wach-Rhythmus-Störungen führen und verschiedene physiologische Prozesse provozieren, die Erkrankungen des kardiovaskulären, metabolischen, endokrinen oder Immunsystems, sowie kognitive oder affektive Störungen verursachen können.

Eine seltene, bei vollständig blinden Menschen aber häufige zirkadiane Rhythmusstörung ist die zyklische Non-24-Schlaf-Wach-Rhythmus-Störung - kurz Non-24 genannt. Bei bis zu $70 \%$ der Blinden mit Restvisus von weniger als $2 \%$ kann es aufgrund der fehlenden Lichtempfindung zur Asynchro-
\end{abstract}

nität zwischen innerem Taktgeber und äußerem Zeitgeber kommen, wie Prof. Peter Young, Direktor der Klinik für Schlafmedizin und Neuromuskuläre Erkrankungen, Universitätsklinikum Münster, berichtete. Die Diagnose erfolgt über Schlafanamnese, Schlaftagebuch und Fragebogen, dazu kommen eine Aktigrafie und ggf. eine Polysomnografie sowie die Melatoninbestimmung.

\section{Gute Synchronisierung}

Für die medikamentöse Therapie steht Tasimelteon (Hetlioz ${ }^{\circ}$ ) zur Verfügung. Die dauerhafte Gabe des Melatonin-Rezeptoragonisten kann einen freien Non-24-Rhythmus bei Blinden synchronisieren, wie die Studien SET und RESET [Lockley SW et al. Lancet. 2015;386:1754-64] zeigten. In den Monaten 1,2 und 7 waren $20 \%, 37 \%$ und
$59 \%$ der Studienteilnehmer mit dem 24-Stunden-Tag synchronisiert. Der Nachtschlaf nahm zu (57 Minuten vs. 17 Minuten unter Placebo), der Tagesschlaf ab (47 Minuten vs. 18 Minuten). Responder behielten unter einer fortgesetzten Therapie zu 90\% die Synchronisierung bei, während sie bei unter Placebo zu $80 \%$ verloren ging.

Als häufigste Nebenwirkungen der Therapie mit Tasimelteon wurden Kopfschmerzen (17\% vs. $7 \%$ ), erhöhte Leberenzyme ( $10 \%$ vs. $5 \%)$ und Alb- bzw. anomale Träume (10\% vs. $0 \%$ ) beobachtet. Resteffekte oder Schläfrigkeit am nächsten Tag sowie relevante endokrine Effekte, Entzugserscheinungen oder erhöhte Suizidalität zeigten sich nicht.

\section{Michael Koczorek}

- Symposium „Die innere Uhr-Einfluss auf Wohlbefinden und Gesundheit", 25. Kongress der Deutschen Gesellschaft für Schlafforschung und Schlafmedizin (DGSM); Münster, November 2017 (Veranstalter: Vanda)

\section{COPD}

\section{LAMA/LABA hat die Nase vorn}

- Für COPD-Patienten der Gruppe „,viele Symptome, wenige Exazerbationen “ empfiehlt das internationale GOLDKomitee die Behandlung mit lang wirksamen Bronchodilatatoren unter Verzicht auf inhalative Steroide (ICS). Die offene CRYSTAL-Studie bestätigt dies. Rekrutiert wurden Patienten mit moderater COPD, die entweder unter Monotherapie mit einem lang wirksamen $\beta_{2}$-Agonisten (LABA) oder einem langwirksamen Anticholinergikum (LAMA) hoch symptomatisch waren (Gruppe A) oder kombiniert mit ICS/LABA behandelt wurden (Gruppe B).

Beide Gruppen wurden 3:1 randomisiert und erhielten entweder die bisherige Therapie weiter oder wurden auf die LABA/LAMA-Fixkombination Indacaterol/Glycopyrronium (Ultibro ${ }^{\circ}$ ) umge- stellt. Die Auswertung nach 120 Tagen Studienlaufzeit ergab, dass die Patienten in beiden Gruppen besser fuhren, wenn sie auf Indacaterol/Glycopyrronium umgestellt wurden, berichtete Studienleiter Prof. Claus Vogel, Marburg.

Das gilt sowohl für die Lungenfunktion, gemessen an der $\mathrm{FEV}_{1}$, als auch für das Kardinalsymptom Atemnot, das anhand des Transition-Dyspnea-Index TDI überprüft wurde. „Das Ergebnis stützt ganz klar die GOLD-Empfehlung, bei symptomatischen Patienten ohne oder mit wenigen Exazerbationen auf Bronchodilatation zu setzen und nicht auf Entzündungshemmung", so Vogelmeier.

Selbst bei Patienten mit hoher Symptomlast und häufigen Exazerbationen (GOLD-Gruppe D) hat LABA/LAMA die Nase vorn, wie die FLAME-Studie

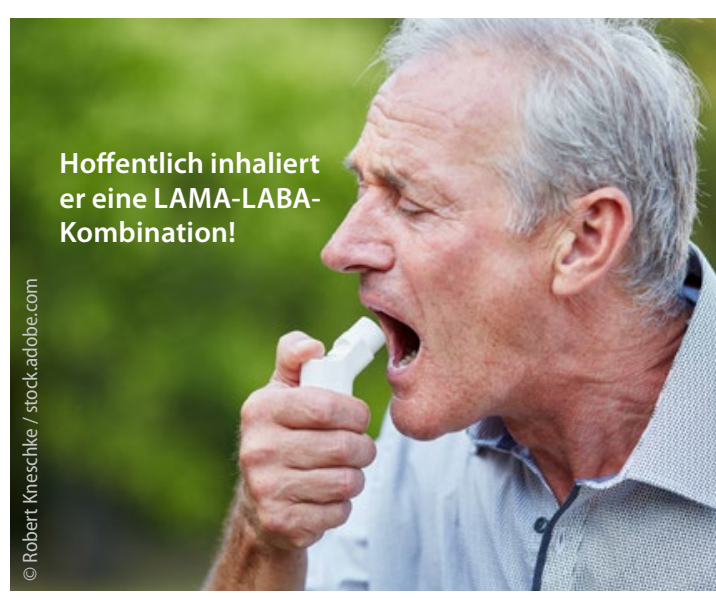

belegt hat. Daher empfiehlt GOLD auch hier die duale Bronchodilatation als Erstlinienoption und ICS nur für Patienten, die unter LABA/LAMA weiter exazerbieren.

\section{Manuela Arand}

- Pressekonferenz „Pneumologie Update - Therapiemanagement bei COPD und Asthma", Kongress der European Respiratory Society; Mailand, September 2017 (Veranstalter: Novartis) 